\title{
Variability of the rs333 in Polish patients with lupus erythematosus
}

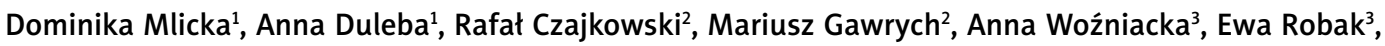 \\ Marta Gorzkiewicz', Szymon Kozłowski ${ }^{4}$, Tomasz Grzybowski ${ }^{1}$, Katarzyna Skonieczna ${ }^{1}$
}

\author{
'Department of Forensic Medicine, Ludwik Rydygier Collegium Medicum in Bydgoszcz, Nicolaus Copernicus University in Torun, Poland \\ 2Department of Dermatology and Venereology, Ludwik Rydygier Collegium Medicum in Bydgoszcz, Nicolaus Copernicus University \\ in Torun, Poland \\ ${ }^{3}$ Department of Dermatology and Venereology, Medical University of Lodz, Lodz, Poland \\ ${ }^{4}$ Independent researcher
}

Adv Dermatol Allergol 2021; XXXVIII (1): 131-136 DOI: https://doi.org/10.5114/ada.2021.104288

\begin{abstract}
Introduction: Lupus erythematosus (LE) is an autoimmune disease with a strong influence of genetic and environmental factors. C-C motif chemokine receptor 5 (CCR5) gene expression may affect the development and intensity of LE. Aim: To evaluate the possible association between the 32bp deletion in rs333 locus located within the CCR5 gene and the development of LE or the occurrence of various clinical symptoms in the course of the disease.

Material and methods: One hundred and twenty patients with LE (77 with systemic lupus erythematosus (SLE) and 43 with discoid lupus erythematosus (DLE)) and 100 healthy controls from the Polish population were genotyped for deletion in rs333.

Results: 32 bp deletion in the rs333 was significantly more frequent among healthy individuals than DLE patients. Moreover, heterozygotes and homozygotes with deletion in rs333 were significantly more frequent within the control group than the group of patients with discoid lupus erythematosus. In contrast, any statistically significant differences in allele or genotype frequencies between healthy persons and SLE patients were observed. Furthermore, nucleotide sequence variability of rs333 was not associated with certain clinical symptoms of LE patients. Conclusions: Deletion in the rs333 might be a protective factor for DLE, but not SLE in the Polish population. Nevertheless further studies performed on larger populations are needed to confirm these observations.
\end{abstract}

Key words: lupus erythematosus, systemic lupus erythematosus, discoid lupus erythematosus, CCR5 gene, rs333.

\section{Introduction}

Lupus erythematosus (LE) is a complex autoimmune disorder with still unclear aetiology. The disease is recognized worldwide, but the frequency, symptoms or fatality depends on ethnic origin [1, 2] as well as age and gender [3-5]. It is worth noting that ethnicity also influences the genetic profile of the illness [6-8]. The clinical picture in the course of LE is very diverse, therefore several subtypes have been identified. Lupus erythematosus may involve only changes within the skin but may also take a systemic form. The most common form of cutaneous LE is discoid lupus erythematosus (DLE). The most se- vere form of LE is systemic lupus erythematosus (SLE), in which the functioning of many internal organs is disturbed. Epidemiological data indicate that about 15\% of DLE patients are progressive to SLE $[9,10]$. It should be noted that the clinicopathological profile of skin lesions is practically identical in both LE subtypes [11, 12] and inflammation is a crucial element in their development [13]. Numerous factors are involved in the pathogenesis of the chronic inflammatory process, including chemokines and their receptors [14-17]. As far as the latter are concerned, one of the most widely studied receptor is C-C chemokine receptor 5 (CCR5).

Address for correspondence: Katarzyna Skonieczna PhD, DSc, Department of Forensic Medicine, Faculty of Medicine, Ludwik Rydygier Collegium Medicum, Nicolaus Copernicus University, 9 Sklodowskiej-Curie St, 85-094 Bydgoszcz, Poland, phone: +48 525853552 , fax: +48 5258535 53, e-mail: k.skonieczna@gmail.com Received: 16.06.2020, accepted: 25.09.2020. 
The CCR5 receptor occurs on the surface of specific cells in the immune system (e.g. T lymphocytes and dendritic cells [18]). Ligands for CCR5 receptors are C-C chemokines. Binding of chemokine molecules by the CCR5 receptor activates $T$ lymphocytes and leads to development of inflammatory reaction in the body [18]. Expression of the CCR5 gene can modify the susceptibility, progression and intensity of many inflammatory diseases [19], including LE. The presence of a functional CCR5 receptor can be associated with the occurrence of inflammation in patients with LE. The deletion of 32 base pairs in the CCR5 gene (in rs333 locus) results in a nonfunctional receptor [20-23]. While some studies suggest that this deletion may have a protective effect on the development and progression of LE [24-28], the other show the lack of association [29-34]. In human populations the allele with deletion occurs with different frequency depending on biogeographic origin. In the European population its frequency is estimated at about $11 \%$, while in the African and Asian populations it does not exceed 1\% [35]. Although the CCR5 receptor is associated with the appearance of inflammation, it is still unclear whether the variability of rs333 is associated with LE development. Taken together, these observations indicate the need for further research into CCR5 gene variability of LE patients and controls of the same biogeographic ancestry.

\section{Aim}

The aim of this study was to analyse nucleotide sequence variability of the rs333 in the Polish population. We also evaluated the possible association between the

Table 1. Clinical characteristics of Polish LE patients

\begin{tabular}{lcc}
\hline Feature & $\begin{array}{c}\text { DLE } \\
n(\%)\end{array}$ & $\begin{array}{c}\text { SLE } \\
n(\%)\end{array}$ \\
\hline Women & $26(60.5)$ & $69(89.6)$ \\
\hline Malar rash & $19(44.2)$ & $60(77.9)$ \\
\hline Discoid rash & $34(79.1)$ & $10(13.0)$ \\
\hline Photosensitivity & $36(83.7)$ & $73(94.8)$ \\
\hline Oral ulcers & $8(18.6)$ & $21(27.3)$ \\
\hline Arthritis & $12(27.9)$ & $33(42.9)$ \\
\hline Pleuritis & $1(2.3)$ & $3(3.9)$ \\
\hline Proteinuria or presence of urine & $6(14.0)$ & $20(26.0)$ \\
crystal formation & $11(25.6)$ & $17(22.1)$ \\
\hline Convulsions or psychosis & $10(23.3)$ & $48(62.3)$ \\
\hline Anaemia with leukopenia, & & \\
\hline lymphopenia or thrombocytopenia & $28(65.1)$ & $76(98.7)$ \\
\hline Raised antinuclear antibodies value & $0(0.00)$ & $2(2.6)$ \\
\hline Spleen disorders & $23(53.5)$ & $59(76.6)$ \\
\hline Joint pain & $12(27.9)$ & $44(57.1)$ \\
\hline Raynaud syndrome & & \\
\hline
\end{tabular}

presence/absence of $32 \mathrm{bp}$ deletion in this gene and the development of DLE and/or SLE. Furthermore, we investigated whether the occurrence of various clinical symptoms is related to the mutation in rs333.

\section{Material and methods}

The research was approved by the Local Ethics Committee of the Nicolaus Copernicus University in Torun, Poland (statement no. KB 562/2013). The study included 120 patients from the Polish population: 77 with SLE and 43 with DLE. SLE was diagnosed according to the guidelines of the American Rheumatological Society updated in 1997 [36], while DLE was diagnosed based on the guidelines indicated by Giliam et al. [37]. Clinical characteristics of Polish patients are summarized in Table 1. The control blood samples were taken from 100 healthy Poles without autoimmune diseases and/or cancer.

Genomic DNA was extracted from peripheral blood (100 individuals from the control group and 60 patients with SLE and 31 patients with DLE) or cheek swabs (17 patients with SLE and 12 patients with DLE) using GeneMATRIX Bio-Trace DNA Purification Kit (EURX, Gdansk, Poland) according to the manufacturer's protocol. The CCR5 gene was amplified by polymerase chain reaction (PCR) using primers developed by Rector et al. [38]. PCR products of $238 \mathrm{bp}$ and $206 \mathrm{bp}$ were analysed by electrophoresis in $3 \%$ agarose gels and stained with ethidium bromide. To check the accuracy of the amplification, PCR products from 2 persons were selected (one from a homozygote with deletion and the other for a homozygote without deletion) and sequenced using dideoxy method. Sequencing reactions were performed with BigDye ${ }^{\oplus}$ Terminator v3.1 Cycle Sequencing Kit (Life Technologies) according to the manufacturer's protocol. PCR and sequencing reactions were performed using GeneAmp PCR System 9700 (Applied Biosystems). 3130xl Genetic Analyzer (Applied Biosystems) was used for capillary electrophoresis. Obtained sequences of the CCR5 gene fragment were compared with the respective reference sequence (GeneBank accession number: NG_012637.1) [39] using the SeqScape v. 2.5 software (Applied Biosystems).

The conformity of the obtained genotypes with Hardy-Weinberg Equilibrium (HWE) was checked using Arlequin software v. 3.5.2.2 [40]. Statistical differences in allele frequencies and genotypes between individuals with diagnosed SLE and/or DLE as well as the control group were examined using $\chi^{2}$ or $\chi^{2}$ tests with Yates correction (Statistica software v. 7.1, StatSoft, Inc., USA). Associations between the clinical traits of patients and the obtained genotypes and alleles were studied with the $\chi^{2}$ test with Yates correction or the Fisher's exact test. In the latter case, the control group consisted of patients with LE, but not showing a given clinical symptom, while the study group included patients with these attributes. Symptoms that occurred in less than $5 \%$ or more than 
95\% of patients with LE (raised antinuclear antibodies value or spleen disorders and pleuritic) were excluded from the analysis.

\section{Results}

Rs333, located within the CCR5 gene, was successfully genotyped in all patients with LE as well as in healthy subjects. Population samples of both cases and controls were found to be in Hardy-Weinberg equilibrium. The allele and genotype frequencies in all groups are given in Table 2.

\section{Associations of rs333 polymorphisms with LE}

The allele with deletion in rs333 was significantly less frequent in the group of all LE (SLE and DLE) patients than in healthy controls ( $p=0.0346$, odds ratio, $\mathrm{OR}=$ 2.0455 with 95\% confidence interval (CI): 1.0149-4.0157). The power of the $\chi^{2}$ test used (with correction for continuity) was approximately 25.0\%. Meanwhile, differences between LE patients and healthy controls were not significantly different for any genotype.

\section{Associations of rs333 polymorphisms with SLE}

There was no statistically significant difference in the allele or genotype frequencies of rs333 between SLE patients and healthy individuals.

\section{Associations of rs333 polymorphisms with DLE}

The allele with deletion in rs333 was statistically less frequent among the DLE patients in comparison to healthy persons ( $p=0.0171, \mathrm{OR}=5.7273$ with $95 \% \mathrm{Cl}$ : $1.3225-24.8037$ ). The power of the $\chi^{2}$ test used (with correction for continuity) was approximately $28.7 \%$. Moreover it was found that homozygotes without deletion were statistically more frequent in DLE patients than in healthy individuals ( $p=0.0214, \mathrm{OR}=0.1729$ with $95 \% \mathrm{Cl}$ : 0.0387-0.772; the test power with continuity correction was approximately 66.8\%). Heterozygotes occurred about three times less frequently in DLE patients than in healthy controls, and the results were statistically significant $(p=0.0375$; OR $=5.125$ with 95\% Cl: 1.1418-23.0039; the power of the test with continuity correction being $55.7 \%)$. There were no statistically significant differences in the prevalence of homozygotes with deletion between the DLE patients and the control group.

\section{Associations of rs333 polymorphisms with clinical features}

No statistically significant differences were revealed in the allele or genotype frequencies of rs333 between patients with SLE and/or DLE with and without specific clinical features (Supplementary Tables S1-S10).

\section{Discussion}

In this study, rs333 located within the CCR5 gene was genotyped in 100 healthy individuals and 120 patients with diagnosed LE (including 77 with SLE and 43 with DLE) of the same biogeographic origin. The investigated population of healthy individuals was in Hardy-Weinberg equilibrium, which indicates the correctness of the performed genotyping and random selection of people from the control group. About 12\% frequency of the allele with deletion in rs333 was revealed in the Polish healthy persons; the value being consistent with the data available in current databases [40-42]. Indeed, the deletion allele occurs in about $11 \%$ of Europeans included in the "1000 Genomes Project" [41]. It is worth noting that in populations outside Europe, this allele occurs with a frequency below 3\% (about 3\% in people of unknown ethnic origin living in America, about $0.9 \%$ in the population of South Asia, about $0.3 \%$ in Africa and virtually no occurrence in East Asia). A similar distribution of allele frequencies appears in the ALFRED database [42], including the Polish population (11.7\% of the deletion allele), which is consistent with the results of this study.

Careful analysis of the data available in population databases and published papers reveals some relationships between the incidence of SLE and the distribution of the rs333 genotypes in subjects of different ethnicities. In general, higher frequencies of the deletion allele are usually accompanied by lower prevalence of SLE. For example, in the Swedish population, the frequency of the allele with deletion is relatively high (about 14\%) [42], while the SLE

Table 2. Associations between rs333 and the occurrence of lupus erythematosus in the Polish population. $P$ - values are given for the $\chi^{2}$ test with Yates correction; WT - allele without deletion in rs333; DEL - allele with deletion in rs333; ${ }^{*} p$-values for the $\chi^{2}$ test

\begin{tabular}{|c|c|c|c|c|c|c|c|}
\hline Allele/genotype & $\begin{array}{c}\text { Controls } \\
N(\%)\end{array}$ & $\begin{array}{c}\text { SLE } \\
N(\%)\end{array}$ & $P$-value & $\begin{array}{c}\text { DLE } \\
N(\%)\end{array}$ & $P$-value & $\begin{array}{c}\text { LE (SLE + DLE) } \\
N(\%)\end{array}$ & $P$-value \\
\hline WT & $176(88.0)$ & 141 (91.6) & \multirow[t]{2}{*}{0.3630} & $84(97.7)$ & \multirow[t]{2}{*}{0.0171} & 225 (93.8) & \multirow[t]{2}{*}{$0.0346^{*}$} \\
\hline DEL & 24 (12.0) & $13(8.4)$ & & $2(2.3)$ & & $15(6.2)$ & \\
\hline WT/WT & 78 (78.0) & $65(84.4)$ & 0.3779 & 41 (95.3) & 0.0214 & $106(88.3)$ & $1.0000^{\star}$ \\
\hline WT/DEL & $20(20.0)$ & $11(14.3)$ & 0.4283 & $2(4.7)$ & 0.0375 & $13(10.8)$ & $1.0000^{*}$ \\
\hline DEL/DEL & $2(2.0)$ & $1(1.3)$ & 0.8189 & $0(0.0)$ & 0.8749 & $1(0.8)$ & 0.8735 \\
\hline
\end{tabular}


prevalence is relatively low (55-65 cases per 100 000) [5]. Similarly, frequency of the deletion allele in Estonians of $14.4 \%$ [42] corresponds to the SLE prevalence of 37-40 cases per 100000 [5]. On the other hand, a higher prevalence of SLE (103 cases per 100 000) was observed in the population of the United Arab Emirates [5], characterized by a relatively low frequency of the deletion allele (about 5\%) [42]. These observations may suggest a protective effect of the rs333 deletion on the development of SLE.

Meanwhile, association data presented in this study showed that allele with deletion in rs333 was significantly less frequent in all patients with LE than in the control population ( $p=0.0346)$. Simultaneously, it was found that the occurrence of this mutation has no significant impact on the development and progression of SLE $(p>0.05)$.

Actually, there are conflicting reports as to how the deletion within the CCR5 gene affects the SLE development. While some studies showed its protective effect for disease propensity $[18,24]$, other reports demonstrated no influence [43-47] or even an increased risk of developing SLE $[25,26]$.

Carvalho et al. [18] examined 219 patients with SLE and 205 healthy individuals from the same region of Portugal. They noted a lower frequency of heterozygotes in SLE patients than in healthy controls and suggested a protective effect of deletion in rs333 on SLE development. A similar relationship was revealed by Schauren et al. [24] among European-derived patients in Brazil. In the same study, 87 patients with SLE and 200 healthy individuals of African origin were examined and no association between the development of SLE and the occurrence of a specific allele was observed. This would suggest the role of biogeographic background in the association of the rs333 variability and the susceptibility to SLE. On the other hand, the lack of association was also reported in patients from Poland [43], Iran [44] the Netherlands [45] and Spain [46]; the observation being consistent with that made in this study. Conversely, the association was revealed in a mixed cohort of patients from Ohio, Colombia and San Antonio (Texas) [26] as well as in a female sample from Brazil [25]. Overall, in the light of all these findings, the issue of possible association between the rs333 locus and the development of SLE remains unresolved and requires further research.

SLE is a multiorgan disease with many different clinical manifestations. Therefore, some groups also analysed the effect of deletion in the rs333 on the development of symptoms in the course of SLE. Carvalho et al. [18] noted absence of homozygotes with deletion in the patient's group with nephritis, central and peripheral nervous system and arthritis involvement. Several studies did not exclude an association of lupus nephritis with the deletion allele [24, 26, 45, 46]. Meanwhile, in the study of Heydarifard et al. [44] no significant association between the rs333 status and clinical features of SLE was revealed.
In this study, no statistically significant associations between the symptoms (or lack thereof) in patients with SLE and/or DLE were found with any allele or genotype.

Until now, no rs333 association studies have been conducted in DLE patients. In this research, it was observed that the occurrence of deletion $32 \mathrm{bp}$ in the CCR5 gene may be a protective factor reducing the risk of DLE development. In fact, the allele with deletion in rs333 was about three times less frequent in DLE patients than in the control group ( $p=0.0171)$. It was also observed that the homozygote without deletion was significantly more frequent in DLE patients than in healthy individuals ( $p=0.0214$ ). Moreover, it was found that the heterozygote was about three times less frequent in DLE patients than in the control group ( $p=0.0375)$.

CCR5 is a chemokine receptor expressed on various cell types, including Th1 lymphocytes, macrophages, fibroblasts, endothelial cells or smooth muscles [18]. The presence of a functional CCR5 receptor may be associated with the initiation and/or maintenance of inflammation in patients with LE. Deletion of $32 \mathrm{bp}$ in the CCR5 gene [18] results in a shorter, non-functional protein. CCR5 gene expression is reduced in heterozygotes and absent in homozygotes with deletion. Inactivated CCR5 receptor is not able of binding chemokines, thus blocking inflammation [25]. Patients with active SLE showed an increased expression of CCR5 receptors on CD4+Th1 cells compared to patients in remission or healthy controls [47]. This would suggest a significant contribution of CCR5 to inflammatory processes in the progression of this disease.

An increased expression of genes associated with the signal pathway activated by $\gamma$ interferon, and consequently, increased activity of Th1 lymphocytes was previously observed in DLE patients [48-50]. Inactivation of the chemokine receptor could play an important role in the prevention of DLE formation. Thus, the presence of deletion in rs333 could be a protective factor against DLE, as the results of this study consistently suggest.

To our knowledge, this is the first study suggesting that the variability of the CCR5 gene sequence may have an impact on DLE development in individuals of Central European descent. However, uncertainties resulting from the power of the implemented statistical tests $(<66.8 \%)$ indicate the need for examining larger groups of patients and healthy controls (at least 166 individuals per group) from biogeographically homogenous populations.

\section{Acknowledgments}

The study was supported by the Nicolaus Copernicus University (grant no. MN-4/WL/2015).

\section{Conflict of interest}

The authors declare no conflict of interest. 


\section{References}

1. Kumar K, Chambers S, Gordon C. Challenges of ethnicity in SLE. Best Pract Res Clin Rheumatol 2009; 23: 549-61.

2. Kaul A, Gordon C, Crow MK, et al. Systemic lupus erythematosus. Nat Rev Dis Primers 2016; 2: 16039.

3. Rivas-Larrauri F, Yamazaki-Nakashimada MA. Systemic lupus erythematosus: is it one disease? Reumatol Clin 2016; 12: 274-81.

4. Nusbaum JS, Mirza I, Shum J, et al. Sex differences in systemic lupus erythematosus: epidemiology, clinical considerations, and disease pathogenesis. Mayo Clin Proc 2020; 95: 384-94.

5. Stojan G, Petri M. Epidemiology of systemic lupus erythematosus: an update. Curr Opin Rheumatol 2018; 30: 144-50.

6. Harley JB, Alarcón-Riquelme ME, Criswell LA, et al. Genomewide association scan in women with systemic lupus erythematosus identifies susceptibility variants in ITGAM, PXK, KIAA1542 and other loci. Nat Genet 2008; 40: 204-10.

7. Han JW, Zheng HF, Cui Y, et al. Genome-wide association study in a Chinese Han population identifies nine new susceptibility loci for systemic lupus erythematosus. Nat Genet 2009; 41: 1234-7.

8. Piotrowski P, Lianeri M, Wudarski M, et al. Contribution of the R620W polymorphism of protein tyrosine phosphatase non-receptor 22 to systemic lupus erythematosus in Poland. Clin Exp Rheumatol 2008; 26: 1099-102.

9. Wieczorek IT, Propert KJ, Okawa J, et al. Systemic symptoms in the progression of cutaneous to systemic lupus erythematosus. JAMA Dermatol 2014; 150: 291-6.

10. Grönhagen CM, Fored CM, Granath F, Nyberg F. Cutaneous lupus erythematosus and the association with systemic lupus erythematosus: a population-based cohort of 1088 patients in Sweden. Br J Dermatol 2011; 164: 1335-41.

11. Cojocaru M, Cojocaru IM, Silosi I, et al. Manifestations of systemic lupus erythematosus. Maedica 2011; 6: 330-6.

12. Grönhagen CM, Nyberg F. Cutaneous lupus erythematosus: an update. Indian Dermatol Online J 2014; 5: 7-13.

13. da Cruz HLA, Cavalcanti CAJ1, de Azęvedo Silva J, et al. Differential expression of the inflammasome complex genes in systemic lupus erythematosus. Immunogenetics 2020; 72: 217-24.

14. Ghorban K, Dadmanesh M, Hassanshahi G, et al. Is the CCR5 $\triangle 32$ mutation associated with immune system-related diseases? Inflammation 2013; 36: 633-42.

15. Guergnon J, Combadière C. Role of chemokines polymorphisms in diseases. Immunol Lett 2012; 145: 15-22.

16. Szekanecz Z, Kim J, Koch AE. Chemokines and chemokine receptors in rheumatoid arthritis. Semin Immunol 2003; 15: 15-21.

17. Murphy PM, Baggiolini M, Charo LF, et al. International union of pharmacology. XXII nomenclature for chemokine receptors. Pharmacol Rev 2000; 52: 145-76.

18. Carvalho C, Calvisi SL, Leal B, et al. CCR5-Delta32: implications in SLE development. Int I Immunogenet 2014; 41: 236-41.

19. Mack M, Brühl H, Gruber R, et al. Predominance of mononuclear cells expressing the chemokine receptor CCR5 in synovial effusion of patients with different forms of arthritis. Arthritis Rheum 1999; 42: 981-8.

20. Samson M, Libert F, Doranz BJ, et al. Resistance to HIV-1 infection in Caucasian individuals bearing mutant alleles of the CCR-5 chemokine receptor gene. Nature 1996; 382: $722-4$.
21. Salem AH, Farid E, Fadel R, et al. Distribution of four HIV type 1- resistance polymorphisms (CCR5- 32 , CR5-m303, CCR2-64I, and SDF1-3'A) in the Bahraini population. AIDS Res Human Retroviruses 2009; 25: 973-7.

22. Grimaldi R, Shindo N, Acosta A, et al. Prevalence of the CCR5 532 mutation in Brazilian populations and cell susceptibility to HIV-1 infection. Human Genet 2002; 111: 102-4.

23. Liu R, Paxton WA, Choe S. et al. Homozygous defect in HIV-1 coreceptor accounts for resistance of some multiply-exposed individuals to HIV-1 infection. Cell 1996; 86: 367-77.

24. Schauren JS, Marasca JA, Velt TD, et al. CCR5 delta32 in systemic lupus erythematosus: implications for disease susceptibility and outcome in a Brazilian population. Lupus 2013; 22: 802-9.

25. Baltus THL, Kallaur AP, Lozovoy MAB, et al. CCR5 32 (rs333) polymorphism is associated with the susceptibility to systemic lupus erythematosus in female Brazilian patients. Rheumatol Int 2016; 36: 7-15.

26. Mamtani M, Rovin B, Brey R, et al. CCL3L1 gene-containing segmental duplications and polymorphisms in CCR5 affect risk of systemic lupus erythematosus. Ann Rheum Dis 2008; 67: 1076-83.

27. Pokorny V, Mcqueen F, Yeoman S, et al. Evidence for negative association of the chemokine receptor CCR5 $\mathrm{d} 32$ polymorphism with rheumatoid arthritis. Ann Rheum Dis 2005; 64: 487-90.

28. Rossol M, Pierer M, Arnold S, et al. Negative association of the chemokine receptor CCR5 d32 polymorphism with systemic inflammatory response, extra-articular symptoms and joint erosion in rheumatoid arthritis. Arthritis Res Ther 2009; 11: R91.

29. Scheibel I, Veit T, Neves AG, et al. Differential CCR5Delta32 allelic frequencies in juvenile idiopathic arthritis subtypes: evidence for different regulatory roles of CCR5 in rheumatological diseases. Scand I Rheumatol 2008; 37: $13-7$.

30. Kohem CL, Brenol JC, Xavier RM, et al. The chemokine receptor CCR5 genetic polymorphism and expression in rheumatoid arthritis patients. Scand J Rheumatol 2007; 36: 359-64.

31. Martens HA, Gross S, Steege G, et al. Lack of association of C-C chemokine receptor $5 \Delta 32$ deletion status with rheumatoid arthritis, systemic lupus erythematosus, lupus nephritis, and disease severity. J Rheumatol 2010; 37: 2226-31.

32. Zapico I, Coto E, Rodriguez A, et al. CCR5 (chemokine receptor-5) DNA-polymorphism influences the severity of rheumatoid arthritis. Genes Immun 2000; 1: 288-9.

33. Aguilar F, Núnes-Roldán A, Torres B, et al. Chemokine receptor CCR2/CCR5 polymorphism in Spanish patients with systemic lupus erythematosus. J Rheumatol 2003; 30: 1770-4.

34. Martens HA, Kallenberg CG, Bijl M. Role of CCR5 Delta32 bp deletion in RA and SLE. Autoimmunity 2009; 42: 260-2.

35. https://www.ncbi.nlm.nih.gov/snp/

36. Hochberg MC. Updating the American College of Rheumatology revised criteria for the classification of systemic lupus erythematosus. Arthritis Rheumatol 1997; 40: 1725.

37. Gilliam JN, Sontheimer RD. Distinctive cutaneous subsets in the spectrum of lupus erythematosus. J Am Acad Dermatol 1981; 4: 471-5.

38. Rector A, Vermeire S, Thoelen I, et al. Analysis of the CC chemokine receptor 5 (CCR5) delta-32 polymorphism in inflammatory bowel disease. Hum Genet 2001; 108: 190-3.

39. https://www.ncbi.n/m.nih.gov/nuccore/NG_012637.1/

40. Excoffier L, Lischer HE. Arlequin suite ver 3.5: a new series of programs to perform population genetics analyses under Linux and Windows. Mol Ecol Resour 2010; 10: 564-7. 
41. https://www.ncbi.nlm.nih.gov/variation/tools/1000genomes/

42. Osier MV, Cheung KH, Kidd JR, et al. ALFRED: an allele frequency database for diverse populations and DNA polymorphisms: an update. Nucleic Acids Res 2001; 29: 317-9.

43. Rzeszotarska E, Sowinska A, Stypinska B, et al. The role of MECP2 and CCR5 polymorphisms on the development and course of systemic lupus erythematosus. Biomolecules 2020; 10: 494.

44. Heydarifard Z, Tabarraei A, Abdollahi N, et al. Evaluation of CCR5 532 polymorphism in patients with systemic lupus erythematosus and healthy individuals. Med Labor I 2018; 12: 38-43.

45. Martens HA, Gross S, van der Steege G, et al. Lack of association of $\mathrm{C}-\mathrm{C}$ chemokine receptor $5 \Delta 32$ deletion status with rheumatoid arthritis, systemic lupus erythematosus, lupus nephritis, and disease severity. J Rheumatol 2010; 37: 2226-31.

46. Aguilar F, Nunez-Roldan A, Torres B, et al. Chemokine receptor CCR2/CCR5 polymorphism in Spanish patients with systemic lupus erythematosus. I Rheumatol 2003; 30: 1770-4.

47. Al-Saleh J, el-Eissawy S. The role of T helper cell subsets in pathogenesis of systemic lupus erythematosus and their relation to disease activity. Egypt J Immunol 2006; 13: 41-8.

48. Crow MK. Type I interferon in the pathogenesis of lupus. J Immunol 2014; 192: 5459-68.

49. Jabbari A, Suárez-Farińas M, Fuentes-Duculan J, et al. Dominant Th1 and minimal Th17 skewing in discoid lupus revealed by transcriptomic comparison with psoriasis. J Invest Dermatol 2014; 134: 87-95.

50. Braunstein I, Klein R, Okawa J, Werth VP. The interferon regulated gene signature is elevated in subacute cutaneous lupus erythematosus and discoid lupus erythematosus and correlates with the cutaneous lupus area and severity index score. Br J Dermatol 2012; 166: 971-5. 